\title{
Review of: "Potential cross-protection against SARS- CoV-2 from previous exposure to bovine coronavirus"
}

\author{
Lorenzo Fraile $^{1}$ \\ 1 Universitat de Lleida
}

Potential competing interests: The author(s) declared that no potential competing interests exist.

This paper tries to address the potential cross-protection against SARS-CoV-2 from previous exposure to bovine coronavirus. The topic is interesting enough to be tackled by authors.

They carried out basically an in silico study to find out common epitopes between bovine coronavirus and SARS-CoV-2 and they hypothetize that persons in contact with bovines should have less clinical impact after a SARS-CoV-2 infection. The data provided are totally speculative without any sound data to support any potential cross-protection.

It should be carried out the following experiments to go on with this research paper:

1.- To study the humoral and cellular immunity for SARS-CoV-2 in humans without previous SARS-CoV-2 infection but with contact with bovines as experimental group.

2.- To study the humoral and cellular immunity in humans for SARS-CoV-2 without previous SARS-CoV-2 infection and without contact with bovines as negative control.

3.- To study the humoral and cellular immunity in humans for SARS-CoV-2 with previous SARS-CoV-2 infection and without contact with bovines as positive control group.

In conclusion, it is necessary to carry out plenty of work to go on with this research work. Anyway, I believe that it is necessary more studies in this line but with a sound scientific design. 